\title{
High- $Q$ Supercavity Modes in Subwavelength Dielectric Resonators
}

\author{
Mikhail V. Rybin, ${ }^{1,2,{ }^{*}}$ Kirill L. Koshelev, ${ }^{1,2}$ Zarina F. Sadrieva, ${ }^{2}$ Kirill B. Samusev, ${ }^{1,2}$ Andrey A. Bogdanov, ${ }^{1,2}$ \\ Mikhail F. Limonov, ${ }^{1,2}$ and Yuri S. Kivshar ${ }^{2,3}$ \\ ${ }^{1}$ Ioffe Institute, St. Petersburg 194021, Russia \\ ${ }^{2}$ ITMO University, St. Petersburg 197101, Russia \\ ${ }^{3}$ Nonlinear Physics Center, Australian National University, Canberra, Australian Capital Territory 2601, Australia
}

(Received 6 June 2017; published 13 December 2017)

\begin{abstract}
Recent progress in nanoscale optical physics is associated with the development of a new branch of nanophotonics exploring strong Mie resonances in dielectric nanoparticles with a high refractive index. The high-index resonant dielectric nanostructures form building blocks for novel photonic metadevices with low losses and advanced functionalities. However, unlike extensively studied cavities in photonic crystals, such dielectric resonators demonstrate low quality factors ( $Q$ factors). Here, we uncover a novel mechanism for achieving giant $Q$ factors of subwavelength nanoscale resonators by realizing the regime of bound states in the continuum. In contrast to the previously suggested multilayer structures with zero permittivity, we reveal strong mode coupling and Fano resonances in homogeneous high-index dielectric finite-length nanorods resulting in high- $Q$ factors at the nanoscale. Thus, high-index dielectric resonators represent the simplest example of nanophotonic supercavities, expanding substantially the range of applications of all-dielectric resonant nanophotonics and meta-optics.
\end{abstract}

DOI: 10.1103/PhysRevLett.119.243901

The trapping of light in localized modes is extremely important for various applications in photonics including lasing [1], sensing [2,3], harmonic generation [4,5], Raman scattering [6], and photovoltaics [7,8]. For many optical devices, it becomes critical to localize electromagnetic fields in subwavelength volumes. Plasmonic structures based on metals allow the subwavelength localization of light by means of surface plasmon polaritons [9]. However, metals impose inevitable losses, which limit the device performance and efficiency. In contrast, high-index dielectric nanoparticles offer a novel way for the subwavelength localization of light due to the Mie resonances being limited only by radiation damping [10]. Unlike metallic nanoscale structures, dielectric nanoparticles support both electric and magnetic Mie modes that expand substantially the applications of meta-optics [11]. Also, dielectric materials with a high refractive index are available in a broad spectral range. At the same time, the standard Mie theory predicts relatively low values of the quality factor ( $Q \approx 5-10)$ for nanoparticles made of conventional optical materials such as $\mathrm{Si}, \mathrm{Ge}$, and $\mathrm{AlGaAs}$, in visible and nearIR spectral ranges.

However, for many applications of all-dielectric nanophotonics, it is very desirable to achieve higher values of the $Q$ factor. One way to enhance the $Q$ factor is to increase the size of the resonator, for example, by confining waves by cavities in photonic crystals [12] or by exploiting whispering gallery modes (WGMs) [13] in disks and high-order Fabry-Pérot modes in elongated rods [14]. Another way is to arrange several resonators in space and excite collective modes [15,16]. An alternative approach for enhancing the $Q$ factors is to use the anapole mode with the spectrally overlapped electric and toroidal dipole modes $[17,18]$. As a result, the $Q$ factor of the anapole mode may approach $Q \approx 30$ [19]. Here we suggest a novel approach based on the concepts of bound states in the continuum and supercavity modes.

The physics of bound states in the continuum (BIC) with a potentially infinite lifetime attracted a lot of attention in photonics [20-23]. Albeit true BIC can exist in structures that are infinitely extended at least at one of the spatial dimensions [24], finite-size samples can support their analogue in the form of supercavity modes [25], with the $Q$ factor growing rapidly before reaching a maximum value limited by the finite-size effects. In accord with the Friedrich-Wintgen theory of BIC [26], the radiating tails of leaky modes can cancel out each other via destructive interference resulting in rapid growth of the $Q$ factor. As an alternative to spatial infinity, one can consider a subwavelength core-shell particle made of material with either infinite or zero permittivity [27,28]; however, for optical frequencies, such materials are not yet common. The earlier studies of high- $Q$ microcavities in the regime of avoided resonance crossing [29] can also be linked to the BIC concept [26]. In this way, a pair of strongly coupled modes of a microcavity may interfere destructively outside the resonator [29] realizing the BIC conditions. Recently, it was shown that even a simple waveguiding system can support BIC by employing an anisotropic material and vectorial nature of electromagnetic fields [30]. It is crucially important that the modes with mixed polarizations may allow a substantial reduction of the resonator's 


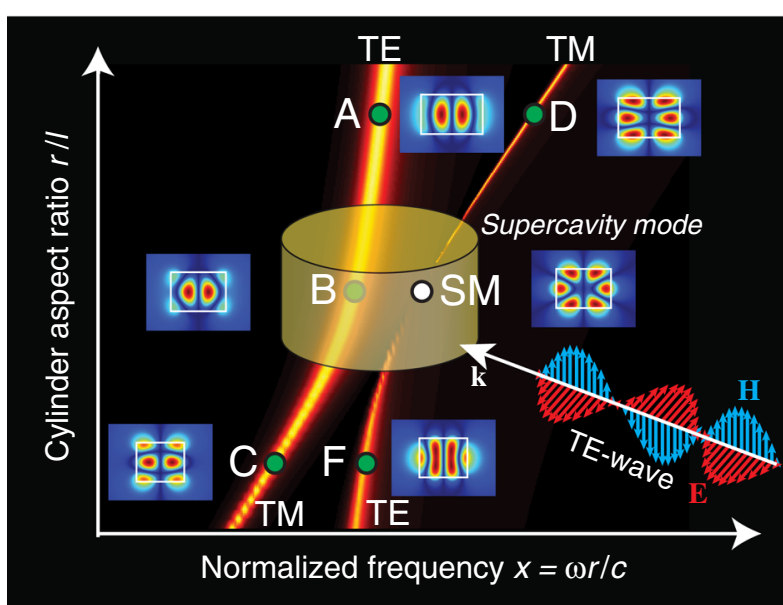

FIG. 1. Illustration of strong mode coupling and a bound state in the continuum supported by a high-index dielectric resonator. The frequency of the TE (Mie-type) mode is a vertical line connecting points $A$ and $F$. When the nanorod aspect ratio varies, this line crosses with the frequency of the TM (Fabry-Pérot-type) mode that connects points $D$ and $C$. This avoided crossing behavior is accompanied by the formation of the supercavity mode with mixed polarization at the SM point. The curve thickness corresponds to the linewidth in the scattering spectra. Insets show the field patterns at marked solid circles.

dimensions, and they can be engineered to realize the supercavity regime [31].

In this Letter, we reveal that subwavelength dielectric resonators with a high refractive index can support the supercavity modes with unexpectedly high $Q$ factors. For an illustrative case of a single dielectric cylindrical resonator, this occurs when two eigenmodes with different polarizations, associated with the Mie resonances and Fabry-Pérot resonances of a finite-length nanorod [32], strongly interact near the avoided crossing regime (Fig. 1). Because these modes are approximately orthogonal inside the resonator, they interfere predominantly outside, realizing the so-called bound states in the continuum and supercavity regime [25] when the subwavelength dielectric resonators demonstrate high values of the $Q$ factor.

We calculate the scattering cross section of the dielectric finite-extent cylindrical resonator as a function of its aspect ratio $r / l$. Here we analyze the modes excited by a TEpolarized plane wave; however, similar effects are observed in the case of TM waves. For generality, we use the normalized size parameter $x=k r$ being a product of the wave number $k$ and resonator radius $r$. To reveal the strong mode coupling effects, we start by setting a high value of the resonator permittivity $\varepsilon=80$. The color map shown in Fig. 2(a) exhibits two families of resonances with the linear dependencies on the aspect ratio $r / l$. This includes the slow-varying TE-polarized (Mie-like) modes mapped to the Mie resonances of an infinitely long cylinder [see Fig. 2(b)] and rapidly varying (Fabry-Pérot-like) modes with the $\mathrm{TM}$ and TE polarization. Here, we employ the standard

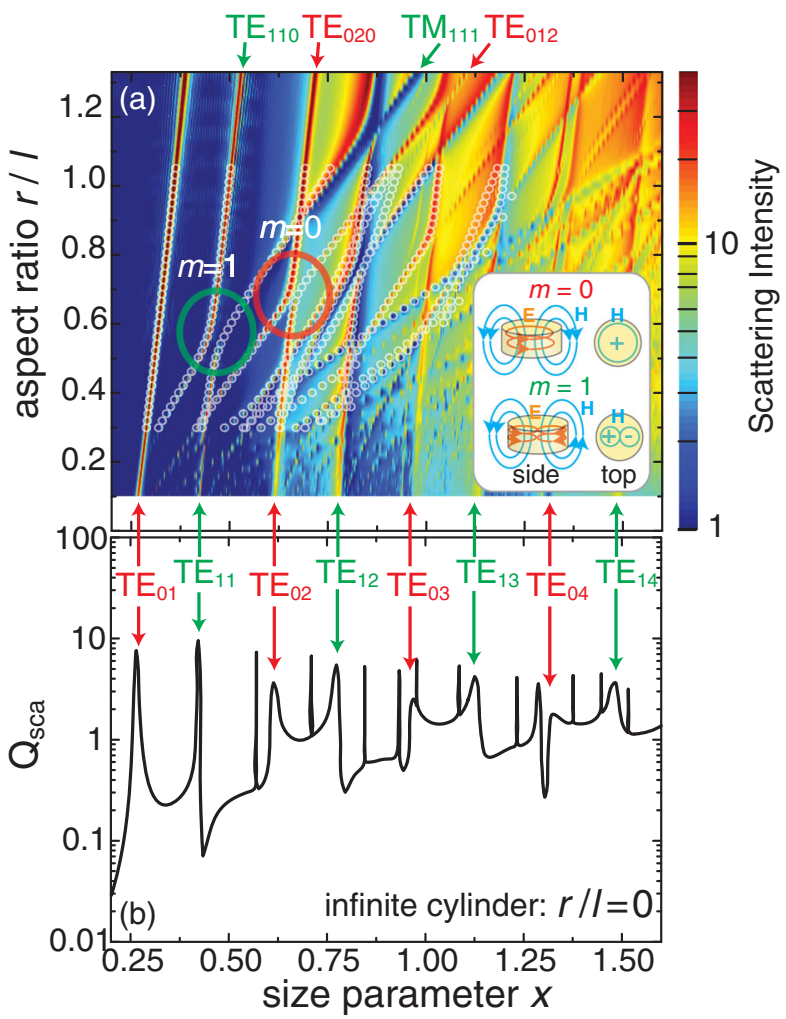

FIG. 2. Dispersion of modes in a high-index dielectric resonator. (a) Plane-wave scattering spectra of a dielectric nanorod with $\varepsilon=80$ vs the (radius-to-height) aspect ratio. Nearly vertical $\mathrm{TE}_{m k n}$ modes correspond to the Mie resonances of an infinite nanowire [with the spectrum shown in (b)] that can be calculated by a rigorous Mie theory. The avoided crossing of the modes with the azimuthal numbers $m=0$ and $m=1$ are marked by red and green circles, respectively. Real parts of the eigenfrequencies are obtained by the resonant-state expansion method and are shown by small circles. The inset in (a) illustrates electric and magnetic fields for the modes with different azimuthal numbers $m$.

mode classification [33], however we have to notice that both TE and TM modes are neither pure transverse electric or magnetic modes but they have a mixed polarization. The first index $m$ corresponds to the azimuthal number of the mode, whereas the other two indices $k$ and $n$ enumerate the Mie and Fabry-Pérot resonances, respectively. Because of different spectral shifts of the Mie and Fabry-Pérot modes when the aspect ratio varies, the modes can intersect at certain points [as shown by red and green circles in Fig. 2(a); other high- $m$ narrow modes in Fig. 2(b) demonstrate similar effects]. Only the modes with the same azimuthal index $m$ could interact, and they undergo coupling each other with the avoided crossing scenario at special values of the aspect ratio parameter $r / l$ with a nearly complete disappearance of spectral lines (see the supercavity mode (SM) point in Fig. 1). We notice that a similar spectral feature was observed in the reflectance spectra of a photonic-crystal membrane supporting BIC [23]. 

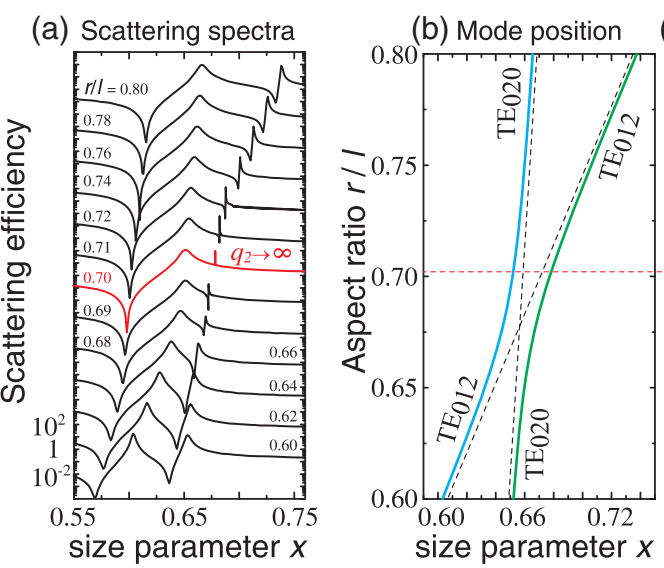

(c) Peak asymmetry
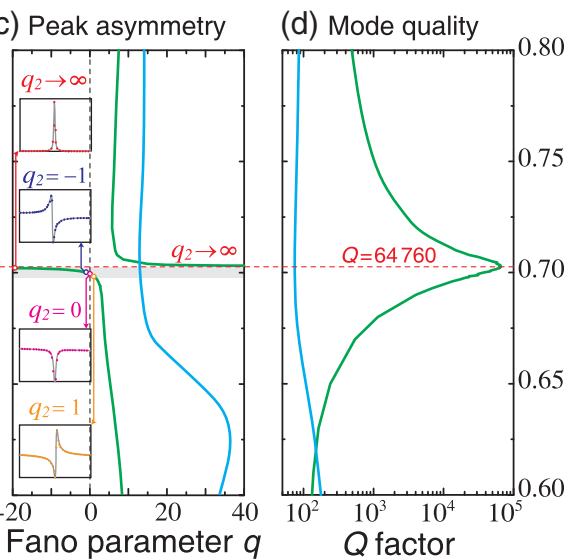

FIG. 3. Characterization of the supercavity modes supported by a dielectric nanoscale resonator. (a) Scattering spectra of a nanorod with $\varepsilon=80$ under the condition of the supercavity mode $(m=0)$. The supercavity mode occurs at $r / l=0.701$ (shown in red). Spectra are relatively shifted by $100 \mathrm{~dB}$. (b) Frequencies of the Mie-type $\mathrm{TE}_{020}$ and Fabry-Pérot-type TE $\mathrm{T}_{012}$ modes in the avoided crossing regime for the modes with the azimuthal number $m=0$. (c) Fano parameter for both modes. The insets show higher-frequency spectral features in the linear scale for the values in a narrow range of $r / l$ marked by orange, magenta, blue, and red circles $(r / l=0.698, q=1$; $r / l=0.699, q=0 ; r / l=0.700, q=-1$; and $r / l=0.703, q \rightarrow \infty)$. (d) Calculated $Q$ factor for a typical supercavity with the maximum close to $Q=65 \times 10^{3}$. Data in (b)-(d) are obtained by fitting to the Fano formula. Cyan and green curves correspond to the low- and high-frequency modes, respectively.

Figure 3 shows the spectra corresponding to the avoided crossing that occurs for $\mathrm{TE}_{020}$ (Mie-like) and $\mathrm{TE}_{012}$ (FabryPérot-like) modes of the dielectric nanoresonator. In the scattering spectra [see Fig. 3(a)], each mode is manifested by an asymmetrical Fano line shape. We study the spectra in detail through the line characteristics such as the central position, damping (proportional to the inverse value of the $Q$ factor), and Fano asymmetry parameter calculated with the help of the classical Fano formula [34,35]. Outside the avoided crossing regime, the frequency shifts of both Mie and Fabry-Pérot modes are described by linear relations [see Figs. 2(a) and 3(b)]. For shorter resonators, when $r / l>0.72$, the spectrum exhibits a pair of asymmetric resonances. The lower-frequency feature corresponds to the excitation of the $\mathrm{TE}_{020}$ (Mie-like) mode at $x=0.66$, and the higher-frequency mode is the $\mathrm{TE}_{012}$ (Fabry-Pérot-type) mode at $x=0.73$. For longer resonators, when $r / l<0.65$, the sequence of the modes is reversed: The Fabry-Pérot mode appears at $x=0.60$, while the Mie-type mode appears at almost the same position $x=0.64$. For $0.65<r / l<0.72$, the modes undergo a strong coupling accompanied by a typical avoided crossing.

Since the modes correspond to asymmetric Fano-like line shapes, the damping parameter cannot be determined directly from the linewidth, but it can be calculated together with the Fano parameter $q$ by fitting to the Fano formula. The dependence of these two parameters on the resonator aspect ratio $r / l$ is shown in Figs. 3(c) and 3(d). For small values of the aspect ratio $(r / l<0.62)$, both modes are characterized by positive $q$ and comparable damping parameters. When the aspect ratio increases, the characteristics of the lower-frequency mode do not vary substantially. In contrast, for the high-frequency mode, both the parameters change dramatically, demonstrating the resonant features in a narrow range of the aspect ratio values, $0.69<r / l<0.71$. At $r / l=0.703$, the asymmetry parameter tends to infinity, and then it becomes negative. The insets in Fig. 3(c) show a change of the scattering spectra in a small range of the aspect ratio values, $0.698<r / l<0.703$. At the same time, the $Q$ factor exhibits a typical dependence of the BIC state [24,25], with the maximum value $Q=65 \times 10^{3}$ limited by finitesize effects.

We notice that the extreme values of both damping and Fano parameters are realized at $r / l=0.703$. In the weakcoupling regime, the Fano asymmetry parameter is known to correspond to the damping rate $[35,36]$, and the case $|q| \rightarrow \infty$ corresponds to the uncoupling of the resonance from the continuum [37]. Hence, we expect similar features also in the case of the strong-coupling regime, and that suggests that the Fano parameter may be employed for the analysis of specific properties of the BIC states. Indeed, the relation between the Fano resonance and BIC was discussed earlier in the context of electronic transport [38], and the limit $|q| \rightarrow \infty$ corresponding to the BIC condition was termed as "collapse of Fano resonance." Also, the asymmetric Fano line shape was identified in the reflection spectra from a photonic-crystal membrane sustaining BIC [23]. Here, we show that an infinite value of the Fano parameter corresponds to BIC or a supercavity mode.

To verify that the effective damping of the higherfrequency mode indeed vanishes, we calculate eigenfrequencies of the resonator. We employ a rigorous method of resonant-state expansion [39]. The real parts of calculated eigenfrequencies are added to Fig. 2(a). Their positions are in excellent agreement with the features in the scattering 
spectra. The analysis of the imaginary parts of the resonator eigenfrequencies confirms that in the vicinity of the avoided crossing point the radiation losses of one mode decrease substantially, revealing its BIC nature. In particular, for $\varepsilon=80$ the $Q$ factor of the supercavity mode is found to be 63700 , being in an agreement with the results obtained from a fitting of the scattering spectra.

Next, we discuss the conditions of the supercavity regime and BIC states in dielectric resonators. For the values of permittivity from the interval $4 \leq \varepsilon \leq 100$, we calculate the resonator eigenvalues by means of the resonant-state expansion method [39] as functions of the aspect ratio $r / l$. We focus on two anticrossing regions marked by circles in Fig. 2(a) for the azimuthal numbers $m=0$ and $m=1$, respectively. For each value of permittivity, the $Q$ factor behaves similar to the dependence presented in Fig. 3(d). The maximum value of $Q$ (in the regime of the supercavity mode) vs $\varepsilon$ is shown in Fig. 4(a). For $\varepsilon>10$, this dependence is well approximated by a power law, $Q=0.04 \varepsilon^{3.2}$, for $m=0$, and $Q=0.1 \varepsilon^{2.3}$, for $m=1$. In particular, for a Si nanoresonator with $\varepsilon=13$, the lower-frequency supercavity mode with $m=1 \mathrm{dem}$ onstrates a $Q$ of about 50 that is at least an order of magnitude higher than the $Q$ factor of a $\mathrm{Si}$ sphere $Q=13.4$, for the magnetic dipole mode, and $Q=6$, for the electric dipole mode. In contrast, the supercavity mode with $m=0$ has a $Q$ of about 200, that is sufficient for strong nonlinear effects at the nanoscale [5].

Figure 4(b) demonstrates the sizes of a cylindrical resonator (normalized by the wavelength) as functions of the material permittivity. Four curves correspond to two diameters (circles) and two heights (squares) for the regime of the supercavity mode with azimuthal numbers $m=0$ (red) and $m=1$ (cyan), respectively. Resonators operate in the subwavelength regime at moderate values of $\varepsilon>3.5$. The Si resonators are shown in the insets. Both dimensions are approximately half wavelength, being much smaller than WGM resonators exploiting high-order $m$ modes.

Importantly, since material losses may significantly lower the values of the $Q$ factors [28], we should study the effect of imperfections. Total losses can be defined by a sum of radiation, absorptions, and surface scattering, namely, $Q_{\mathrm{rad}}^{-1}+Q_{\mathrm{abs}}^{-1}+Q_{\mathrm{ss}}^{-1}$. We find that in dielectric nanoresonators the $Q$ factors of supercavity modes are restricted by the quantity $\operatorname{Re}(\varepsilon) / \operatorname{Im}(2 \varepsilon)$. For undoped crystalline silicon, $Q_{\mathrm{abs}}>10^{10}$ for $\lambda>1.5 \mu \mathrm{m}$. Losses due to surface scattering can be estimated as $Q_{\mathrm{ss}} \approx 10^{13}$ [40]. Thus, these two mechanisms are negligible relative to the radiation losses $Q \approx 200$. Additionally, we verify the robustness of the effect under inevitable fluctuations of the geometrical sizes and observe that a change of the resonator diameter $\Delta D<15 \mathrm{~nm}$ changes the $Q$ factor by less than $2 \%$.

We notice that the mode interference associated with the BIC state can be described in terms of the Friedrich-
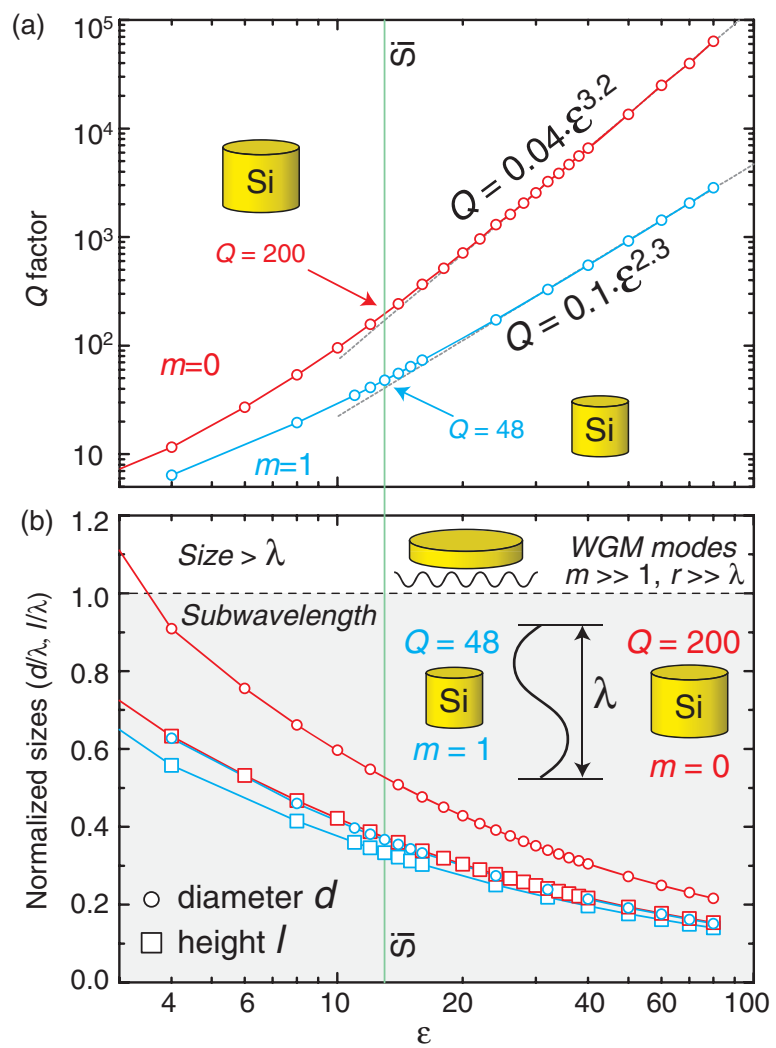

FIG. 4. Calculated $Q$ factors of the supercavity modes realized in subwavelength dielectric resonators. (a) Dependence of the $Q$ factor of the modes with the azimuthal numbers $m=0$ and $m=1$ on the dielectric permittivity. For high values of permittivity, the $Q$ factor demonstrates a power growth. (b) Relative dimensions of a dielectric cylindrical resonator supporting a supercavity mode. All sizes are normalized by the resonant wavelength. A resonator becomes subwavelength below a boundary marked by a horizontal line. Diameters are depicted by circles; heights are shown by squares. The insets illustrate the cases of a silicon resonator for $m=0$ and $m=1$. All data in (a) and (b) are calculated by the resonant-state expansion method. The case of silicon is marked by a vertical line.

Wintgen theory [26] developed earlier for electronic BIC states. This theory assumes that a pair of discrete states interact through the continuum of delocalized states. This interaction shifts the energy levels of the discrete states, and at certain conditions the states undergo a strong mode coupling. As a result, the damping rate of one state increases, while the damping rate of the other state decreases until it vanishes and BIC appears. In photonic systems, a pair of resonator modes can be described by the Friedrich-Wintgen theory provided the size of the optical modes is negligible relative to the wavelength in the media. The calculated damping rate being inverse to the $Q$ factor shown in Fig. 3(d) exhibits a strong suppression (peak in the $Q$ factor) being in excellent agreement with the predictions of the Friedrich-Wintgen theory. When the dielectric permittivity increases, one achieves higher $Q$ 
factors [see Fig. 4(a)], because the modes become close to the theoretical idealization. This theory suggests how to engineer the resonator properties for achieving really high $Q$ factors for simple geometries.

In summary, we have revealed that subwavelength highindex dielectric resonators can support the supercavity modes with high $Q$ factors, provided their parameters are tuned to match the BIC conditions. For Si resonators, our approach suggests $Q \sim 200$, that is sufficient for many applications. We believe that our finding opens new horizons for nanoscale metadevices including lowthreshold nanolasers, biosensors, parametric amplifiers, and nanophotonics quantum circuits.

The authors acknowledge fruitful discussions with A. Alù, W. Barnes, R. W. Boyd, C. De Angelis, A. Fratalocchi, S. Gladyshev, J.S. Totero Gongora, B. Kante, A. A. Kaplyanskii, A. Moroz, J. Sanchez-Gil, and J. Sipe. Theoretical studies have been supported by the Ministry of Education and Science of the Russian Federation (3.1500.2017/4.6), the Russian Foundation for Basic Research (16-02-00461), and the Australian Research Council. Simulations of complex eigenmodes have been supported by the Russian Science Foundation (17-12-01581).

*m.rybin@mail.ioffe.ru

[1] A. Kodigala, T. Lepetit, Q. Gu, B. Bahari, Y. Fainman, and B. Kanté, Nature (London) 541, 196 (2017).

[2] M. C. Gather and S. H. Yun, Nat. Photonics 5, 406 (2011).

[3] M. R. Foreman, J. D. Swaim, and F. Vollmer, Adv. Opt. Photonics 7, 168 (2015).

[4] J. L. Dominguez-Juarez, G. Kozyreff, and J. Martorell, Nat. Commun. 2, 254 (2011).

[5] A. S. Shorokhov et al., Nano Lett. 16, 4857 (2016).

[6] B.-B. Li, W. R. Clements, X.-C. Yu, K. Shi, Q. Gong, and Y.-F. Xiao, Proc. Natl. Acad. Sci. U.S.A. 111, 14657 (2014).

[7] P. Spinelli, M. A. Verschuuren, and A. Polman, Nat. Commun. 3, 692 (2012).

[8] M. L. Brongersma, Y. Cui, and S. Fan, Nat. Mater. 13, 451 (2014).

[9] O. Hess, J. B. Pendry, S. A. Maier, R. F. Oulton, J. M. Hamm, and K. L. Tsakmakidis, Nat. Mater. 11, 573 (2012).

[10] A. I. Kuznetsov, A. E. Miroshnichenko, M. L. Brongersma, Y. S. Kivshar, and B. Luk'yanchuk, Science 354, aag2472 (2016).

[11] Y. Kivshar and A. Miroshnichenko, Opt. Photonics News 28, 24 (2017).

[12] T. Asano, Y. Ochi, Y. Takahashi, K. Kishimoto, and S. Noda, Opt. Express 25, 1769 (2017).

[13] G. C. Righini, Y. Dumeige, P. Féron, M. Ferrari, D. Ristic, and S. Soria, Riv. Nuovo Cimento Soc. Ital. Fis. 34, 435 (2011).

[14] D. R. Abujetas, M. A. Mandujano, E. R. Mendez, and J. A. Sanchez-Gil, ACS Photonics 4, 1814 (2017).
[15] U. Zywietz, M. K. Schmidt, A. B. Evlyukhin, C. Reinhardt, J. Aizpurua, and B. N. Chichkov, ACS Photonics 2, 913 (2015).

[16] M. I. Shalaev, J. Sun, A. Tsukernik, A. Pandey, K. Nikolskiy, and N. M. Litchinitser, Nano Lett. 15, 6261 (2015).

[17] A. A. Basharin, M. Kafesaki, E. N. Economou, C. M. Soukoulis, V. A. Fedotov, V. Savinov, and N. I. Zheludev, Phys. Rev. X 5, 011036 (2015).

[18] A. E. Miroshnichenko, A. B. Evlyukhin, Y. F. Yu, R. M. Bakker, A. Chipouline, A. I. Kuznetsov, B. Luk'yanchuk, B. N. Chichkov, and Y. S. Kivshar, Nat. Commun. 6, 8069 (2015).

[19] G. Grinblat, Y. Li, M. P. Nielsen, R. F. Oulton, and S. A. Maier, ACS Nano 11, 953 (2017).

[20] D. C. Marinica, A. G. Borisov, and S. V. Shabanov, Phys. Rev. Lett. 100, 183902 (2008).

[21] E. N. Bulgakov and A. F. Sadreev, Phys. Rev. B 78, 075105 (2008).

[22] Y. Plotnik, O. Peleg, F. Dreisow, M. Heinrich, S. Nolte, A. Szameit, and M. Segev, Phys. Rev. Lett. 107, 183901 (2011).

[23] C. W. Hsu, B. Zhen, J. Lee, S.-L. Chua, S. G. Johnson, J. D. Joannopoulos, and M. Soljačić, Nature (London) 499, 188 (2013).

[24] C. W. Hsu, B. Zhen, A. D. Stone, J. D. Joannopoulos, and M. Soljačić, Nat. Rev. Mater. 1, 16048 (2016).

[25] M. Rybin and Y. Kivshar, Nature (London) 541, 164 (2017).

[26] H. Friedrich and D. Wintgen, Phys. Rev. A 32, 3231 (1985).

[27] F. Monticone and A. Alu, Phys. Rev. Lett. 112, 213903 (2014).

[28] M. G. Silveirinha, Phys. Rev. A 89, 023813 (2014).

[29] J. Wiersig, Phys. Rev. Lett. 97, 253901 (2006); H. Cao and J. Wiersig, Rev. Mod. Phys. 87, 61 (2015).

[30] J. Gomis-Bresco, D. Artigas, and L. Torner, Nat. Photonics 11, 232 (2017).

[31] Y. Kivshar and M. Rybin, Nat. Photonics 11, 212 (2017).

[32] Y. Yang, A. E. Miroshnichenko, S. V. Kostinski, M. Odit, P. Kapitanova, M. Qiu, and Y. S. Kivshar, Phys. Rev. B 95, 165426 (2017).

[33] K. Zhang and D. Li, Electromagnetic Theory for Microwaves and Optoelectronics (Springer, Berlin, 2008).

[34] M. V. Rybin, A. B. Khanikaev, M. Inoue, K. B. Samusev, M. J. Steel, G. Yushin, and M. F. Limonov, Phys. Rev. Lett. 103, 023901 (2009).

[35] M. F. Limonov, M. V. Rybin, A. N. Poddubny, and Y. Kivshar, Nat. Photonics 11, 543 (2017).

[36] M. V. Rybin, S. F. Mingaleev, M. F. Limonov, and Y. S. Kivshar, Sci. Rep. 6, 20599 (2016).

[37] D. M. Riffe, Phys. Rev. B 84, 064308 (2011).

[38] C. S. Kim, A. M. Satanin, Y. S. Joe, and R. M. Cosby, Phys. Rev. B 60, 10962 (1999).

[39] M. B. Doost, W. Langbein, and E. A. Muljarov, Phys. Rev. A 90, 013834 (2014).

[40] M. Borselli, T. J. Johnson, and O. Painter, Opt. Express 13, 1515 (2005). 